\title{
COVID-19 hypothesis: Exosomes of mesenchymal stem cells as nano-cargos for anti-SARS-CoV-2 asRNAs
}

\author{
Alireza Afshar ${ }^{1}$, Masood Zare ${ }^{1}$, Zohreh Farrar ${ }^{1}$, Alireza Hashemi ${ }^{1}$, Arezoo Khoradmehr ${ }^{1}$, \\ Hassan Habibi ${ }^{2}$, Mohammad Amin Behzadi ${ }^{3}$, and Amin Tamadon ${ }^{1}$ \\ ${ }^{1}$ Boushehr University of Medical Sciences \\ ${ }^{2}$ Persian Gulf University \\ ${ }^{3}$ Icahn School of Medicine at Mount Sinai
}

December 28, 2020

\begin{abstract}
The severe acute respiratory syndrome coronavirus 2 (SARS-CoV-2) emerged in December 2019 is rapidly spreading worldwide. Scientists are searching to find an effective treatment for coronavirus disease 2019 (COVID-19). Several antiviral drugs are currently undergoing clinical trial studies to evaluate their safety and efficacy in the treatment of COVID-19. SARS-CoV-2 is a positive-sense single-stranded RNA virus. Previous studies showed the efficacy of anti-RNA virus, single strand RNA inhibiting antisense RNAs (asRNAs), on silencing of virus replication, in vitro. To transfer the anti-SARS-CoV-2 asRNAs to human respiratory epithelium, exosomes can be suggested as a promising candidate. Mesenchymal stem cells (MSCs) secret exosomes and they can be loaded by anti-RNA virus asRNAs. MSCs-secreted exosomes as a nano-cargo of anti-SARS-CoV-2 asRNAs have other therapeutic potentials such as immunomodulatory effects of their cytokine contents, affinity to respiratory epithelial attachment, anti-fibrotic activity in lung, non-toxicity for normal cells, and do not trigger an immune response. Inhalation of anti-SARS-CoV-2 asRNAs may stop SARS-CoV-2 replication. Producing a specific anti-SARS-CoV-2 asRNAs by targeting the genome of virus and their delivery by MSCs exosomes is suggested and discussed. This approach potentially sheds light on gene therapy of the other human lung diseases via inhalational delivery using exosomes in future.
\end{abstract}

\section{Hosted file}

Afshar et al. COVID-19 treatment hypothesis.pdf available at https://authorea.com/users/ 385769/articles/500991-covid-19-hypothesis-exosomes-of-mesenchymal-stem-cells-as-nano-

cargos-for-anti-sars-cov-2-asrnas 